an $\mathrm{MCO}$ research network (MCORN) or state health department (SHD), we compared the data available to an MCORN to the data available to an SHD Results: Data available to the SHD included hospital discharges, case fatality rates by diagnosis for hospitalized patients, counts of surgical procedures, and death certificate data. The disadvantage of organizing a surveillance system through an SHD is that hospitalizations are not linked to services provided during the hospitalization or survival after discharge. Likewise, behavioral risk factor data, medication data and data on ventricular function of patients with heart disease are not available. While MCOs have these data and data linkages, the disadvantage of organizing a surveillance system through an MCORN is that, although covering a significant segment of the US population, the surveillance system would not be strictly populationbased. Conclusions: The data required to identify clinical opportunities to prevent and postpone deaths are available to a greater extent through MCOs than an SHD. The large populations covered by MCORNs, their geographic range, the relatively stable populations, and the implementation of electronic medical records all make large MCORNs attractive alternatives to SHD surveillance systems.

PS2-33:

Understanding Racial Disparities in Physician Advice and Patient Actions to Control Blood Pressure in an MCO

Douglas W Roblin, PhD, Kaiser Permanente Georgia; Peter J Joski, MSPH, Kaiser Permanente Georgia; Edmund R Becker, PhD, Rollins School of Public Health at Emory University

Background/Objective: The Chronic Care Model (CCM) links proactive practice teams and activated patients with better chronic care outcomes. We studied the associations of blood pressure (BP) control with 1) physician advice and patient actions on lifestyle behaviors (exercise, diet, salt and alcohol intake) to control BP and 2) medication adherence in a cohort of adults with hypertension (HTN) in a managed care organization (MCO). Our primary analysis focused on the apparent paradox that, in this MCO, African Americans were more likely to report receipt of advice and be taking actions to control BP yet had worse BP control. Methods: Adults 18-74 years of age with HTN were identified from computerized data. Two independent samples of 3,000 adults each (750 per JNC-VII level) were randomly selected for telephone survey in October 2007 and March 2008. The survey included BRFSS items on advice and actions to control BP and self-reported race and education. Medication adherence was measured as proportion of days covered (PDC) with any HTN-related medication in the 12-month period preceding the survey. Mean SBP and DBP were measured from computerized data in the same period. Associations among receipt of advice, taking actions, PDC, and BP control were estimated using multivariate path analysis (controlling for age, gender, and education) for the 1,330 respondents who were African American (751) or white (579). Results: Compared to whites, African Americans had significantly $(\mathrm{p}<0.05)$ higher SBP (standardized beta of 0.137). Better medication adherence was associated with lower SBP (0.229); however, African Americans had lower medication adherence (0.074). Patients taking action to control BP were more likely to have received physician advice to take action (0.232); and, African Americans were more likely to receive advice $(0.187)$ and to take action $(0.056)$. Poor SBP was associated with greater likelihood of taking action (0.075). A similar pattern of associations was observed for DBP. Conclusions: Compared to Whites, African Americans in this MCO were less adherent with antihypertensive medications; and, this contributed to worse BP control. Worse BP control, however, increased the likelihood that physicians advised African Americans to take actions to control BP. Consistent with the CCM, physicians appear to be directing their advice on lifestyle actions to the subset of patients most likely to benefit with improved BP control.

\section{Clinical Effectiveness}

C-C3-01:

A Conditional Sequential Sampling Procedure for Drug Safety Surveillance

Lingling Li, PhD, Department of Ambulatory Care and Prevention, Harvard Medical School and Harvard Pilgrim Health Care

Background: Health plans' administrative claims data are known to be very useful in post-marketing drug or vaccine safety surveillance to detect certain adverse events that are difficult to capture during the preapproval clinical trails. To detect the existence of excess risk for an adverse drug event, hypothesis testing should be conducted whenever data is updated and thus appropriate group sequential or sequential analysis methods should be implemented to adjust for multiple testing and preserve the overall type I error rate. Methods: We propose a practical group sequential method, a conditional sequential sampling procedure, to derive valid inference on the parameter of interest, the relative risk for an adverse drug event between the drug of interest and the comparison drug. The method allows the information for both drug groups to be accumulated prospectively and thus, unlike the newly developed maximized sequential probability ratio test (MaxSPRT), doesn't require the availability of a lot of historical data for the comparison drug. Moreover, the method automatically adjusts for population heterogeneity and temporal trend and requires no a priori assumptions on how the baseline incidence rates change across strata and over time. In addition, the method remains valid (i.e., preserves the nominal Type I error rate) even when the number of interim tests is large and/or there are a lot of strata defined based on several potential confounders. We will explain why the standard general group sequential theory method might not be appropriate in such settings. Results: We have conducted an extensive simulation study to evaluate the performance of the new method and compare that to the performance of the general group sequential theory method in a subset of scenarios when applicable. The power performance for both methods in the considered scenarios is very similar and our new method applies to much more general settings. Conclusions: We will also implement this method to the data collected in the HMO Research Network CERT II study. This method can be particularly useful in prospective drug surveillance studies in which both drugs are relative new and not enough historical data is available.

\section{C-C3-02:}

Increasingly Restrictive Definitions of Hyperkalemia Outcomes in a Database Study: Effect on Incidence Estimates

Marsha A Raebel, PharmD, Kaiser Permanente Colorado; Colleen Ross, MS, Kaiser Permanente Colorado; Leslie A Wright, MA, Kaiser Permanente Colorado; Craig Cheetham, PharmD, MPH, Kaiser Permanente Southeast; Hans Peterson, MS, Lovelace Respiratory Research Institute; Douglas W Roblin, PhD, Kaiser Permanente Georgia; David H Smith, PhD, Kaiser Permanente Northwest

Background/Aims: Determination of hyperkalemia associated adverse outcomes incidence and risk assessment is complicated by lack of consistent hyperkalemia definitions across studies. Further, information about hyperkalemia from clinical trials, while reflecting the potential of reninangiotensin-aldosterone system (RAAS) inhibitor treatment to increase serum potassium $(\mathrm{K})$ concentration above a defined level, may not reflect risk or severity of outcomes. We sought to examine to what extent increasing levels of restriction influenced incidence estimates of hyperkalemia outcomes. Methods: The study cohort was drawn from a population of adult patients with diabetes at 3 HMORN sites. We identified all new users of a RAAS inhibitor between 01/01/2001 and 12/31/2006 and assessed hyperkalemiaassociated outcomes within the first year of therapy. The initial definition of a hyperkalemia outcome to which other definitions were compared included any ambulatory (AV), emergency department (ED) or inpatient (IP) visit with a $\mathrm{K}$ level $>5.5 \mathrm{mmol} / \mathrm{l}$ or a coded hyperkalemia diagnosis within 7 days of the visit. The following restrictions were then applied: a) increasing minimum $\mathrm{K}$ concentration to $>6.0 \mathrm{mmol} / \mathrm{l}$; b) reducing timeframe to 24 hours; and c) removing AV. Crude incidence rates of hyperkalemia-associated adverse outcomes were calculated using person years ( $p-y)$ determined as time from drug initiation to first outcome or other censoring event (e.g., drug discontinuation, end of study). Results: 
The cohort included 27,362 patients. Mean duration of initial therapy was 212 days. Hyperkalemia-associated outcome incidence estimates varied from 33.6 per 1000 p-y (defined as AV, ED or IP visit with $\mathrm{K}>5.5$ or a coded hyperkalemia diagnosis within 7 days) to 11.0 per $1000 \mathrm{p}-\mathrm{y}$ (defined as IP or ED visit with $\mathrm{K}>6$ or coded diagnosis within 24 hours). Removing AV had the greatest effect and reducing the timeframe to 24 hours had the least effect on incidence estimates. Conclusions: Modifying hyperkalemia definition criteria resulted in up to a 3-fold difference in by estimates of hyperkalemiaassociated adverse outcomes. Further work linking these findings to adverse events is critical to decisions regarding appropriate definitions to answer specific study questions. We caution against comparing incidence estimates across published studies without considering the severity implications of differences in hyperkalemia outcomes definitions.

\section{C-C3-03:}

From Chart to CART: Improving Automated Case-Finding for Ectopic Pregnancy Using CART Analysis

Onchee Yu, MS, Group Health, Center for Health Studies; Jane Grafton, BA, Group Health, Center for Health Studies; Victoria L Holt, PhD, Dept of Epidemiology, University of Washington; Britton Trabert, PhC, Dept of Epidemiology, University of Washington; Linda Wehnes, BA, Group Health, Center for Health Studies; Patricia Yarbro, MSW, Group Health, Center for Health Studies; Delia Scholes, PhD, Group Health, Center for Health Studies

Background/Aims: Research and surveillance work addressing ectopic pregnancy (EP) rely largely on ICD diagnostic codes and CPT procedure codes available from automated data sources. However, cases identified in this way may not be true EP cases, as these codes may also be used to rule out, follow up or note a history of EP. Through the use of additional automated data on treatment, procedures, and other aspects of care, we developed a classification algorithm that could improve the accuracy of EP case identification. Methods: Using Group Health automated data files, we initially identified 2,632 potential EP episodes occurring in women aged 1544 years during 1988-2007 using ICD and CPT codes for EP and for surgical or laparoscopic treatment of EP. Chart reviews to verify EP status were conducted on 130 potential EP cases in the algorithm development dataset and on 150 in the algorithm validation dataset. Using additional information on demographic factors, other diagnosis and procedure codes, treatment modalities, site of care (ambulatory vs. inpatient), and laboratory data available with each EP episode, we conducted a classification and regression tree (CART) analysis to create a case finding algorithm for EP. Results: From the CART analysis, the case-finding algorithm for EP contained three main predictors: at least two encounter dates with an EP diagnosis or procedure code during an episode; treatment with methotrexate; and presence of an ICD -9 code of $633.1,633.10$ or 633.11 for tubal pregnancy. Sensitivity for the development and validation sets, respectively, was $95 \%$ and $91 \%$ while specificity was $78 \%$ and $83 \%$. The EP misclassification rate using the new algorithm was $10.7 \%$ in the development set and $11.5 \%$ in the validation set compared to $32 \%$ when EP cases were originally defined using EP diagnosis and procedure codes. Conclusions: The CART-derived algorithm for identifying EP cases was highly sensitive, had good specificity, and misclassification rates were notably improved over the original case identification techniques. Additional pharmacy and encounter data available in many health plan databases can markedly improve the accuracy of EP identification. In particular, the identified predictors in the algorithm are available in the CRN VDW, and it would be of interest to test this algorithm at other HMORN sites.

C-C3-04:

Using Electronic Health Record Data to Predict Heart Failure Diagnosis

Nirav R Shah, MD, MPH, Geisinger Center for Health Research; Jason Roy, $\mathrm{PhD}$, Geisinger Center for Health Research; Walter F Stewart, PhD, Geisinger Center for Health Research

Background: Heart Failure (HF) is one of the most common and serious progressive illnesses among elderly patients. It is usually detected at a relatively advanced stage, after irreversible damage has occurred. Early detection offers the potential to substantially reduce patient disability and health care costs. Objective: To develop a novel detection strategy, making use of longitudinal electronic health record (EHR) data to create an HF early detection prediction model. Methods: All data for this study were obtained from Geisinger Clinic's EHR among patients who had a primary care provider. A prediction model was developed using a nested case control study design, where HF cases diagnosed between 2003-2006 were identified and controls were randomly selected matched on sex, age, and clinic. We used conditional logistic regression to model the relation between EHR data and detection of HF $6+$ months and $18+$ months before the actual date of diagnosis. Variables for the model included diagnoses, the most recent lab and clinical (e.g., SBP, DBP, pulse pressure) measures, medication orders and ambulatory care use in the previous two years, and smoking status. Data were only used if they occurred in the record either $6+$ months or $18+$ months before the diagnosis date, depending on the specific model. Model results were validated by combining a bootstrap re-sampling approach with a backwards elimination selection method. Results: We identified at least one matching control for 2,239 of the 2,764 cases; 9 or 10 controls were identified for $81 \%$ of the cases. A total of 24,249 controls were selected. The model for detecting HF $6+$ months before usual diagnosis had an AUC of 0.80 ; the parallel model for detecting HF $18+$ months before usual diagnosis had an AUC of 0.75 (95\% CI: 0.73, 0.79). The AUC findings were similar for separate models completed on systolic HF and diastolic HF. Conclusions: In practice, clinicians do not have the time or ability to process seemingly disparate data points over a series of visits that might suggest a preclinical HF for a given patient. Our analysis of EHR data indicate that HF can be detected 6 or more months before usual diagnosis with good AUC and high specificity. These findings suggest that routine evaluation of EHR data may be useful in screening for patients at high risk of $\mathrm{HF}$, creating numerous opportunities for early and aggressive intervention and potentially altering the natural history of heart failure for many patients.

PS1-04:

Does Mode Matter? A Comparison of Depression Scores from Mail and Phone

Cheryl Wiese, MA, Group Health Center for Health Studies; Paula Sandler, BA, Group Health Center for Health Studies; Julie Richards, MPH, Group Health Center for Health Studies

Background: Researchers often consider mixing modes for data collection to increase response rate. However, it is possible that people respond differently when asked sensitive questions (like questions about depression) by mail compared to phone. Methods: A random sample of 4000 adolescent health plan enrollees (ages 13-17 years) were invited to participate in a brief survey examining exercise, sedentary behaviors, and depressive symptoms. Mailings were directed at parents and included an invitation letter, a consent form, survey, and a $\$ 2$ pre-incentive for the youth. Parents were instructed to complete the consent form and give the survey to their child to complete and return. Non-responders were sent a second survey. Among remaining nonresponders, $60 \%$ of the adults were phoned, asking them to review and sign the consent form and to ask their child to complete the screener. Forty percent of remaining non-responders were phoned asking the adults' permission to complete the screener with the child by phone. In a second study, 9,838 adults with a history of diabetes or heart disease were invited to participate in a randomized controlled trial. The initial contact was a mailed 5-item survey that included a depression (PHQ2) screener and eligibility questions, along with a study information sheet and a $\$ 2$ pre-incentive. Approximately 12 days later, non-responders were sent a second survey, and 10 days later, nonresponders were called to complete the screener by phone. Results: Data collection is being completed as this abstract is submitted. This poster will examine whether there are differences in depression scores among those who are early responders (complete after one mailing), those who complete after a second mailing, and those who complete by telephone. We will also examine whether there are demographic differences at each step. In addition, we will demonstrate the increases in response rate by adding a phone call to complete a mailed survey. Conclusions: This poster will indicate whether this mixing of modes compromises data quality or produces different responses than if only one mode was used. 